\title{
Video Article \\ A Novel Clinical Grade Isolation Method for Human Kidney Perivascular Stromal Cells
}

\author{
Daniëlle G. Leuning ${ }^{1}$, Ellen Lievers ${ }^{1}$, Marlies E.J. Reinders ${ }^{1}$, Cees van Kooten ${ }^{1}$, Marten A. Engelse ${ }^{1}$, Ton J. Rabelink ${ }^{1}$ \\ ${ }^{1}$ Department of Internal Medicine, Leiden University Medical Centre
}

Correspondence to: Ton J. Rabelink at a.j.rabelink@lumc.nl

URL: https://www.jove.com/video/55841

DOI: doi: $10.3791 / 55841$

Keywords: Medicine, Issue 126, Tissue-specific stem cells, stromal cells, mesenchymal stromal cells, mesenchymal stem cells, pericytes, kidney, perivascular stromal cells, renal stem cells, solid organ, collagenase digestion.

Date Published: 8/7/2017

Citation: Leuning, D.G., Lievers, E., Reinders, M.E., van Kooten, C., Engelse, M.A., Rabelink, T.J. A Novel Clinical Grade Isolation Method for Human Kidney Perivascular Stromal Cells. J. Vis. Exp. (126), e55841, doi:10.3791/55841 (2017).

\section{Abstract}

Mesenchymal Stromal Cells (MSCs) are tissue homeostatic and immune modulatory cells that have shown beneficial effects in kidney diseases and transplantation. Perivascular Stromal Cells (PSCs) share characteristics with bone marrow MSCs (bmMSCs). However, they also possess, most likely due to local imprinting, tissue-specific properties and play a role in local tissue homeostasis. This tissue specificity may result in tissue specific repair, also within the human kidney. We previously showed that human kidney PSCs (kPSCs) have enhanced kidney epithelial wound healing whereas bmMSCs did not have this potential. Moreover, kPSCs can ameliorate kidney injury in vivo. Therefore, kPSCs constitute an interesting source for cell therapy, particularly for kidney diseases and renal transplantation. Here we show the detailed isolation and culture method for kPSCs from transplant-grade human kidneys based on whole-organ perfusion of digestive enzymes via the renal artery and enrichment for the perivascular marker NG2. In this way, large cell quantities can be obtained that are suitable for cellular therapy.

\section{Video Link}

The video component of this article can be found at https://www.jove.com/video/55841/

\section{Introduction}

Mesenchymal Stromal Cells (MSCs) are immune modulatory cells that were originally isolated from the bone marrow. They are characterized by their spindle shaped morphology, ability to differentiate into fat, bone and cartilage, and plastic adherence. MSCs express the stromal markers CD73, CD90, CD105 while being negative for the markers CD31 and CD45 ${ }^{1,2,3}$. MSCs are a promising candidate for cell therapy due to their tissue homeostatic and immunomodulatory capacities. bmMSCs are currently being studied in clinical trials for several diseases, including kidney diseases and kidney transplantation as reviewed elsewhere ${ }^{4}$.

Previously it was shown that perivascular cells from several different solid organs, including adipose tissue, placenta and skeletal muscle share characteristics with MSCs ${ }^{9}$. However, these cells also exhibit tissue-specific functions which can result in organotypic repair ${ }^{10}$. Human myocardial perivascular cells, for example, stimulated angiogenic responses after hypoxia and differentiated into cardiomyocytes, while perivascular cells isolated from other tissues did not show these potentials ${ }^{11}$

Perivascular stromal cells can also be isolated from the mouse $\mathrm{e}^{12,13,14}$ and human kidney ${ }^{15,16}$. We extensively characterized kPSCs and compared these to bmMSCs. We found that kPSCs, similar to bmMSCs, have immunosuppressive capacities and can support vascular plexus formation. However, there are tissue specific differences between the cell types, as kPSCs showed an organotypic transcriptional expression signature, including the nephrogenic transcription factors HoxD10 and HoxD11. kPSCs, in contrast to bmMSCs, did not undergo myofibroblast transformation after stimulation with TGF- $\beta$ and were unable to differentiate into adipocytes. Furthermore, kPSCs accelerated epithelial integrity in a kidney tubular epithelial wound scratch assay, a phenomenon which was not observed with bmMSCs. This enhanced wound repair was mediated through hepatocyte growth factor release. Moreover, kPSCs ameliorated kidney injury in a mouse model of acute kidney injury ${ }^{15}$. Therefore, kPSCs seem to have superior renal repair capacities and are an interesting new source for cell therapy in kidney diseases.

In order to be able to use kPSCs for cell therapy purposes, kPSCs should be isolated in a clinical grade manner with clinical grade enzymes and protocols. Moreover, to be able to treat several patients with kPSCs from 1 donor, sufficient cell numbers should be obtained. Here we show in detail, the clinical grade isolation procedure of KPSCs from whole transplant grade kidneys, yielding sufficient numbers of cells to be used for clinical cellular therapy.

\section{Protocol}

The local medical ethical committee and ethical advisory board of the European consortium (STELLAR) approved the research and collection of human transplant grade kidneys discarded mainly for surgical reasons. Research consent was given for all kidneys. 


\section{Preparations for Cell Culture}

1. Prepare a pool of platelet lysates.

1. Store the platelets that have expired for less than $2 \mathrm{~d}$ in $-80^{\circ} \mathrm{C}$ until use (for a maximum of 1 year).

NOTE: The platelets were originally sourced from a commercial vendor. Hospital surplus material distributed by the local blood bank were obtained.

2. Thaw the platelets of at least 5 donors (preferably 10 ) $\mathrm{O} / \mathrm{N}$ at $4{ }^{\circ} \mathrm{C}$.

3. Pool the platelets in a large sterile bottle, transfer to conical centrifuge tubes and spin down for $10 \mathrm{~min}$ at $1,960 \times \mathrm{g}$ at $4{ }^{\circ} \mathrm{C}$. NOTE: The volume of platelets depends on the number of donors and the amount of hospital surplus material per donor.

4. Pipette the supernatant to blood bags $(50 \mathrm{~mL} / \mathrm{bag})$ through the barrel of a $50 \mathrm{~mL}$ syringe. Store at $-80^{\circ} \mathrm{C}$.

2. Preparation of cell culture medium.

1. Defrost one bag of platelet lysates in a water bath at $37^{\circ} \mathrm{C}$.

2. Add $1 \mathrm{~L}$ (i.e. 2 bottles) of Minimum Essential Medium - alpha modification (alphaMEM), $5 \mathrm{~mL} 200 \mathrm{mM}$ glutamine, and $20 \mathrm{~mL}$ of penicillin $(5,000 \mathrm{U} / \mathrm{mL}) /$ streptomycin $(5,000 \mu \mathrm{g} / \mathrm{mL})($ pen/strep $)$ to the bag.

3. Incubate for $3 \mathrm{~h}$ at $37^{\circ} \mathrm{C}$

4. Shake the bag for degelling by gently tapping on the bag when the bag is resting on a flat surface.

5. Filter the $5 \%$ platelet lysates medium by pulling the lysates through the filter of the transfusion system with a sterile $50 \mathrm{~mL}$ syringe.

6. Aliquot the medium in $50 \mathrm{~mL}$ tubes and store in $-80^{\circ} \mathrm{C}$ until use.

NOTE: Every new batch of platelet lysates is tested in cell culture and compared to the previous batch by growing cells of interest (bmMSCs or kPSCs) to confluency in both batches. In cell numbers and viability, the kPSCs or bmMCs grown in the new batch should not differ more than $10 \%$, and the marker expression of CD73, CD90, CD105 and CD31, CD34 and CD45 as analyzed by flow cytometry should not differ. The methods of cell culture are described in section 6 of the protocol (Culture of kPSC).

\section{Preparations for Cell Harvest}

1. Preparation of solutions.

1. Prepare the plain medium by adding $10 \mathrm{~mL}$ of pen/strep to $500 \mathrm{~mL}$ (1 bottle) of Dulbecco's Minimum Essential Medium (DMEM)-F12.

2. Prepare the washing medium by adding $50 \mathrm{~mL}$ of Normal Human Serum (NHS) and $10 \mathrm{~mL}$ of pen/strep to 1 bottle of DMEM-F12.

3. Prepare the enzyme-stock buffer by adding $2.9 \mathrm{~mL} 1 \mathrm{M} \mathrm{4-(2-hydroxyethyl)-1-piperazineethanesulfonic} \mathrm{acid} \mathrm{(HEPES),} 1.2 \mathrm{~mL} \mathrm{NaHCO}$ $2.5 \%[\mathrm{w} / \mathrm{v}]$ and $160 \mu \mathrm{L} \mathrm{CaCl} 2(1 \mathrm{M})$ to $160 \mathrm{~mL}$ University of Wisconsin solution.

4. Prepare the collagenase solution by slowly adding $20 \mathrm{~mL}$ of enzyme stock buffer to the bottle of GMP-grade collagenase containing $>2,000 \mathrm{U} /$ bottle collagenase. Dissolve at $4{ }^{\circ} \mathrm{C}$ for approximately $40 \mathrm{~min}$. Gently shake several times during the dissolving period.

5. Prepare the freeze medium by adding $5 \mathrm{~mL}$ dimethyl sulfoxide (DMSO) to $45 \mathrm{~mL}$ NHS.

2. Preparation of perfusion tray (Figure 1A).

1. Clean the flow cabin and cover with surgical drapes. Heat a water bath to approximately $40-45^{\circ} \mathrm{C}$ to heat the perfusion tray to $37{ }^{\circ} \mathrm{C}$. Put on surgical gowns and surgical gloves.

2. Connect a sterilized perfusion tray to the water bath to preheat the perfusion tray with warm water. Make sure that there is no air in the perfusion tray by starting with the perfusion tray in the vertical position. If necessary add more water to the water bath to prevent air infusion into the perfusion tray.

3. Place the LS25 tube in the pump. Leave both sterile ends of the tube in the flow cabinet. Place a Kocher's forceps on one end of the tube and allow to rest on the bottom of the perfusion tray. Place a sterile gauze on top of the tube to prevent obstruction. Attach a Luer connector on the other tube end.

4. Add approximately $100 \mathrm{~mL}$ of plain medium into the perfusion tray and start flushing the tube on a pump speed of $100 \mathrm{~mL} / \mathrm{min}$.

\section{Kidney Cell Isolation}

1. Preparation of the kidney.

1. Remove the kidney from the double sterile bags and place on the sterile perfusion tray (Figure 1B). Remove the perirenal adipose tissue and kidney capsule with scissors and gentle tearing (Figure 1C) and identify the renal artery on the aortic patch as shown in Figure 1D.

2. Cannulate the renal artery with the accessory spike, fix with a sterilized tie-rip, and attach to the pump tube end with the Luer connector while the pump is on. If the tubes are not completely filled, first add more medium to the tray and flush tubes by starting the pump (Figure 1E - F). Flush the kidney with plain medium via the pump with a flow of $100 \mathrm{~mL} / \mathrm{min}$.

2. Collagenase treatment and kidney cell isolation.

1. Add collagenase $(20 \mathrm{~mL},>2,000 \mathrm{U})$ and DNase $(2.5 \mathrm{~mL}, 1 \mathrm{mg} / \mathrm{mL})$ to the perfusion tray (Figure 1G). Turn the kidney from time to time gently by hand. Wait until the kidney becomes soft and the perfusion liquid becomes less transparent (approximately 30 - 40 min).

2. Gently massage the kidney (Figure 1H) until a cell suspension is obtained (Figure 1I). Remove non-digested material and the spike in the renal artery.

3. Washing and collection of kidney cells.

1. Add $50 \mathrm{~mL}$ of the NHS to the cell suspension. Drain the cell suspension from the perfusion tray by putting the pump at low flow and placing the tube first connected to the kidney above the $50 \mathrm{~mL}$ tubes (Figure 1J). 
2. Centrifuge at $300 \times \mathrm{g}$ for $7 \mathrm{~min}$ at $4{ }^{\circ} \mathrm{C}$ and remove the supernatant. Wash the pellets with washing medium containing $10 \% \mathrm{NHS}$ and again centrifuge at $300 \times \mathrm{g}$ for $7 \mathrm{~min}$. Repeat washing once more. Measure the volume of the cell pellets and add an equal volume of cell culture medium.

3. Either cryopreserve the cells from here by adding freezing medium 1:1 to the cell suspension and store in cryogenic vials according to standard protocols, or continue to culture the cells (section 4).

NOTE: The cell suspension contains cell clumps and requires extra steps to obtain single cells, which results in an increase in cell death. Therefore, the cells are kept in suspension and are not counted at this stage.

\section{Cell Culture of Crude Kidney Cells}

1. Add approximately $1 \mathrm{~mL}$ of the cell suspension to $24 \mathrm{~mL}$ of alphaMEM $5 \%$ platelet lysates (cell culture medium) in a T175 cell culture flask (Figure 1K) and culture at $37^{\circ} \mathrm{C}, 5 \% \mathrm{CO}_{2}$. Remove the medium and cell debris after $2 \mathrm{~d}$ from the adherent cells and refresh the medium with $25 \mathrm{~mL}$ of cell culture medium.

2. Refresh the culture medium twice a week by removing half of the medium (12.5 mL) and adding fresh medium (12.5 mL) using aseptic techniques.

3. Culture until confluent (usually $5-7 \mathrm{~d}$ ) (Figure 1L). Remove the medium, wash twice with PBS and trypsinize the cells by adding $5 \mathrm{~mL}$ trypsin to each T175 flask for $5 \mathrm{~min}$ at $37^{\circ} \mathrm{C}$. Wash in culture medium and centrifuge at $490 \mathrm{xg}$ for $5 \mathrm{~min}$ and remove the supernatant.

\section{Cell Enrichment for NG2 by Magnetic Cell Separation (Figure 1M)}

1. Prepare the cell separation buffer according to manufacturer's protocol.

2. Resuspend the trypsinized kPSCs in $10 \mathrm{~mL}$ cell separation buffer. Centrifuge at $490 \mathrm{xg}$ for $5 \mathrm{~min}$, discard the supernatant and resuspend the cells in $300 \mu \mathrm{L}$ cell separation buffer.

3. Add $100 \mu \mathrm{L} \mathrm{FCR}$ blocking reagen $/ 5 \times 10^{7}$ cells, add $100 \mu \mathrm{L}$ anti-melanoma (NG2) beads per $5 \times 10^{7}$ cells, and incubate for $30 \mathrm{~min}$ at $4{ }^{\circ} \mathrm{C}$. Separate the NG2-positive fraction according to manufacturer's protocol. Add $10 \mathrm{~mL}$ of medium and centrifuge cells for $5 \mathrm{~min}$ at $490 \times \mathrm{g}$.

4. Count the cells using a Bürker counting chamber according to manufacturer's protocol. Seed the cells in a culture flask (approximately $4 \times 10^{3}$ cells $/ \mathrm{cm}^{2}$ ) and incubate.

NOTE: Seed the cells in the following concentrations: $<250,000$ in a T25 flask, 250,000 - 500,000 in a T75 flask, and 700,000 - 800,000 in a T175 flask. After magnetic cell enrichment, a positive fraction of approximately $1-2 \%$ is isolated. However, as this step is cell enrichment and not cell sorting, other cell types will be present in the culture (Figure 1N). After several passages (usually around $4-5$ ), only a homogenous kPSC-population is present (Figure 1P).

\section{Culture of kPSCs}

1. Refresh the medium twice a week by removing half of the medium and replace it with freshly thawed culture medium (Figure $1 \mathrm{~N}$ ). NOTE: The kPSCs tend to be more viable and proliferative when only half of the medium is refreshed.

2. Passaging of kPSCs.

NOTE: Passage the kPSCs at either 90 - 100\% confluency, or when 3D structures appear (see Figure 10), or when there hasn't been cell growth for more than a week. The latter two particularly might occur at early passages after cell enrichment. In this case, after passaging, the cells will usually start to grow again in monolayer culture (Figure 1P).

1. Remove the medium from the $90-100 \%$ confluent cells (keep the medium for later use). Wash the flask twice with PBS (1 mL in T25, $5 \mathrm{~mL}$ in T75, $10 \mathrm{~mL}$ in T175). Add trypsin $\left(0.5 \mathrm{~mL}\right.$ in T25, $2 \mathrm{~mL}$ in T75, $5 \mathrm{~mL}$ in T175) and incubate for $5 \mathrm{~min}$ at $37^{\circ} \mathrm{C}$. Add the old medium to the flask and resuspend gently.

2. Transfer to a 15 or $50 \mathrm{~mL}$ tube (depending on the volume) and centrifuge at $490 \mathrm{xg}$ for $5 \mathrm{~min}$. Count the viable cells and plate $<250,000$ in a T25, 250,000-500,000 in a T75, or 700,000-800,000 in a T175 (approximately $4 \times 10^{3} \mathrm{cells} / \mathrm{cm}^{2}$ ).

3. Test the kPSCs at passage 5 or 6 (e.g., scratch assay, section 7 ).

NOTE: Characterize the propagated cells by flow cytometry using standard protocols. Marker expression of NG2, PDGFR- $\beta$, CD146, CD73, CD90, CD105, HLA-ABC should all be positive and CD31, CD34, CD45 and HLA-DR should be negative. Test for mycoplasma, bacteria and fungi in the culture medium according to standard protocols of the clinical microbiology lab. Test the kPSCs functionally in a kidney epithelial wound scratch assay. Experiments are usually performed with sterile, flow cytometry-confirmed homogeneous kPSC populations with wound healing capacity between passage 6-8.

4. Cryopreserve the $\mathrm{kPSCs}$ by adding $0.5 \mathrm{~mL}$ cryopreservation medium to $0.5 \mathrm{~mL}$ cell suspension ( 2 million cells per $\mathrm{mL}$ of culture medium) using standard protocols.

NOTE: After thawing, seed the kPSCs at a higher density $\left(10^{6}\right.$ cells in a T175).

\section{Functional Test of kPSCs: Kidney Epithelial Wound Scratch Assay}

1. Culture the kPSCs in a 6-well plate at a density of 200,000 cells/well in $2 \mathrm{~mL}$ culture medium. After $48 \mathrm{~h}$ of culture at $37{ }^{\circ} \mathrm{C}, 5 \% \mathrm{CO}_{2}$, collect the supernatant. This is the conditioned medium

2. Seed the HK-2 (proximal tubular epithelial cells) ${ }^{17}$ in $2 \mathrm{~mL}$ of Proximal Tubular Epithelial Cell (PTEC) medium consisting of a $1: 1$ ratio of DMEM-F12 supplemented with insulin $(5 \mathrm{mg} / \mathrm{mL})$, transferrin $(5 \mathrm{mg} / \mathrm{mL})$, selenium $(5 \mathrm{ng} / \mathrm{mL})$, hydrocortisone $(36 \mathrm{ng} / \mathrm{mL})$, triiodothyrinine $(40$ $\mathrm{pg} / \mathrm{mL})$, epidermal growth factor $(10 \mathrm{ng} / \mathrm{mL})$ and pen/strep, in a density of 500,000 cells per well in a 6 -well cell culture plate.

3. Culture for $48 \mathrm{~h}$ at $37{ }^{\circ} \mathrm{C}, 5 \% \mathrm{CO}_{2}$. Remove the cell culture medium of the HK-2s and create a scratch wound in the monolayer of HK-2s by making a scratch with the tip of a yellow pipette point from the top to the bottom of the well. Wash the HK-2s with PBS and add the conditioned medium of the kPSCs or control medium. Mark on the bottom of the plate the area to be imaged. NOTE: The HK-2s should be confluent. Image two areas per well. 
4. Image the scratch at $4,8,12$ and $24 \mathrm{~h}$ at the same marked position with an inverted bright-field microscope.

5. Measure the scratch area in Image $\mathrm{J}$ using the polygon selection tool to determine the percentage of closure.

\section{Representative Results}

The clinical grade kPSCs isolation method is summarized in Figure 1. Crude kidney cells are isolated from human transplant grade kidneys by collagenase perfusion. The resulting cell suspension is cultured until confluent in $5 \%$ platelet lysates. Then the perivascular stromal cell fraction is isolated based on NG2 expression.

kPSCs are plastic adherent spindle-shaped cells (Figure 2A) and are positive for the stromal markers CD73, CD90, CD105 and perivascular markers NG2, PDGFR-B and CD146, while negative for CD31, CD34, CD45 and HLA-DR (Figure 2B). Typically, a homogeneous population of kPSCs can be reached at passage 4 and kPSCs reach senescence around passage 9 - 10 (Figure 2C). We therefore advise to perform experiments between passage 5 - 8 .

To evaluate the functional capacity of the isolated kPSCs, we perform an in vitro kidney epithelial wound scratch assay on every new batch of KPSCs, as we previously showed that the conditioned medium of kPSCs can accelerate epithelial wound healing in this scratch assay ${ }^{15}$. The kPSC conditioned medium is made by culturing kPSCs for $48 \mathrm{~h}$ in alphaMEM $5 \%$ platelet lysates and collecting the supernatant. Next, the immortalized human kidney proximal tubule epithelial cells (HK-2) ${ }^{17}$ are cultured until confluent and then a scratch wound is made. Then either the conditioned medium of KPSCs or control medium is added to the wells and the speed of wound healing is measured. When the conditioned medium of kPSCs is added, the wound closes significantly faster (Figure 2D).
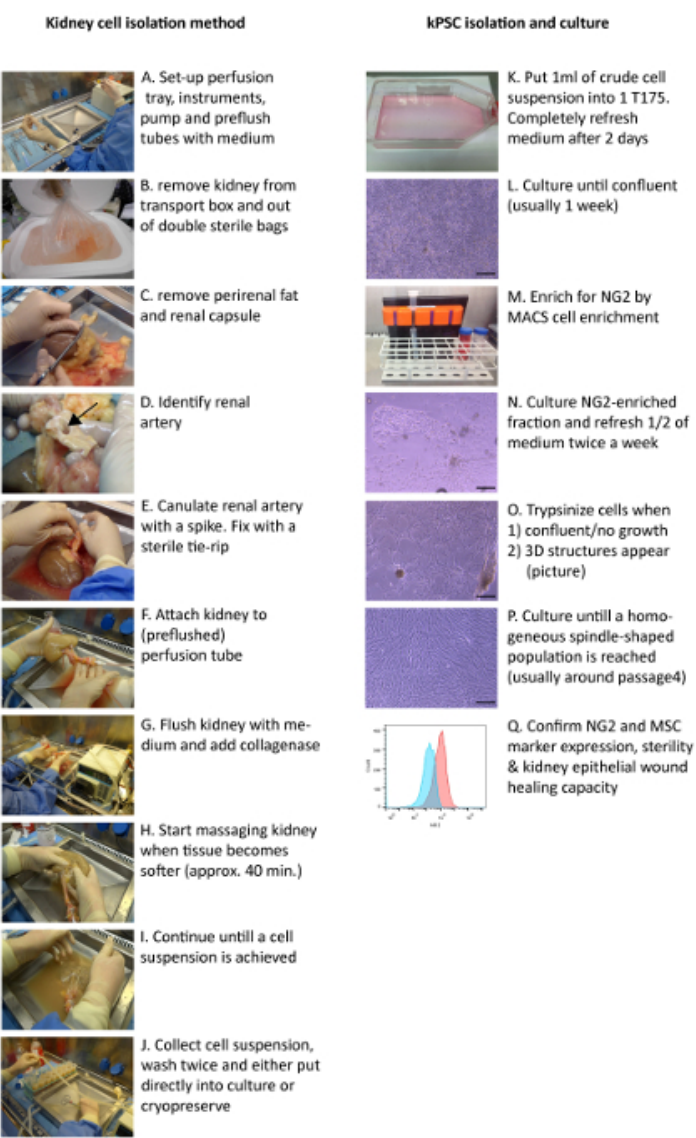

Figure 1: Clinical Grade Isolation Method of Human kPSCs. Transplant grade kidneys are cannulated and perfused with collagenase via the renal artery $(\mathbf{A}-\mathbf{G})$ and the resulting cell suspension is washed and either cryopreserved or put into culture $(\mathbf{H}-\mathbf{K})$. After the cells reach confluency (L), NG2 cell enrichment is performed (M). Cells are trypsinized when they either are confluent, have stopped proliferating or when 3D structures appear $(\mathbf{N}-\mathbf{P})$. The release criteria for KPSCs are sterility, marker expression and the capability to enhance tubular epithelial wound healing $(\mathbf{Q})$. Arrow: renal artery. Scale bar $=200 \mu \mathrm{m}$. Please click here to view a larger version of this figure. 
A

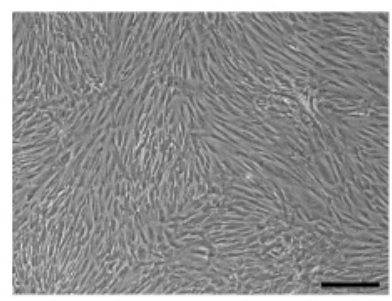

C

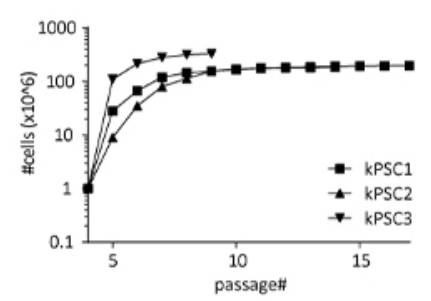

D

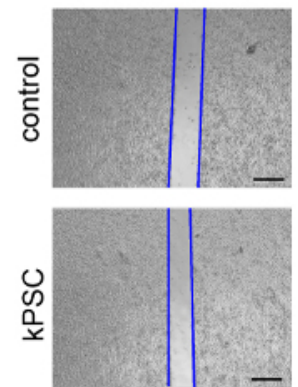

$\mathrm{t}=0$
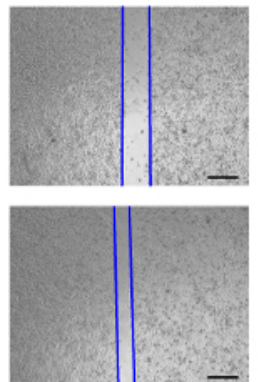

$t=4$
B
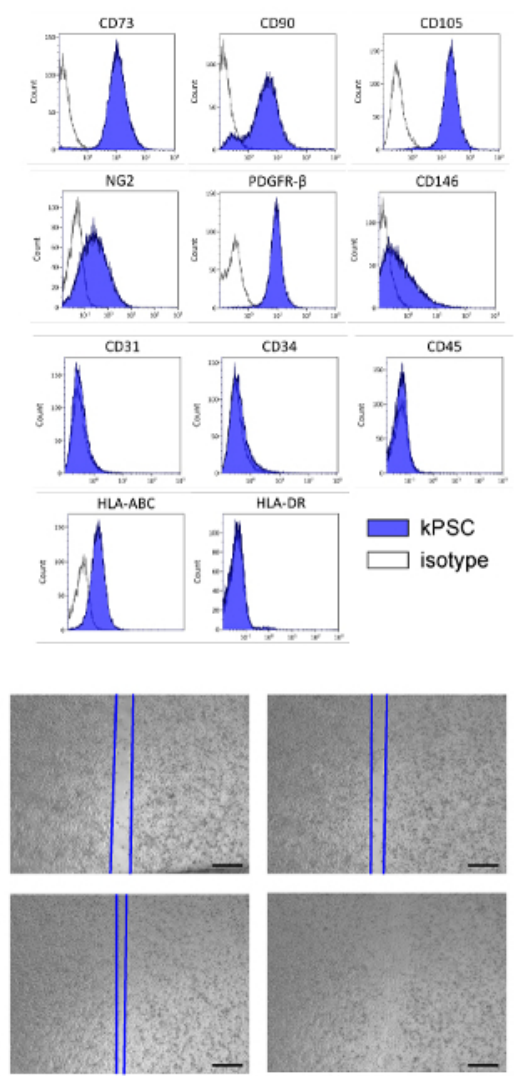

$t=8$

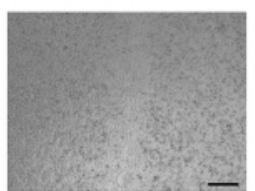

$t=12$

Figure 2: Characterization of Human kPSCs. A) kPSCs are spindle-shaped, plastic adherent cells. B) kPSCs are positive for mesenchymal markers CD73, CD90, CD105, perivascular markers NG2, PDGFR- $\beta$ and CD146, while negative for CD31, CD34 and CD45. kPSCs express MHC-class I (HLA-ABC) but not class II (HLA-DR). C) Growth characteristics of three different kPSC donors from flow cytometry confirmed homogeneous NG2 positive populations (at passage 4). kPSCs reach senescence around passage $9-10$. D) kPSCs are able to enhance kidney epithelial repair in a wound scratch assay. Representative images of control medium and $\mathrm{kPSC}$ conditioned medium at $\mathrm{t}=0,4,8$ and $12 \mathrm{~h}$ are shown. Scale bar in $A)=200 \mu \mathrm{m}$, in $D)=100 \mu \mathrm{m}$. Please click here to view a larger version of this figure.

\section{Discussion}

Perivascular cells have been isolated from many different human solid organs, including pancreas, fat, cartilage and the kidney ${ }^{9,15,16}$. Most methods, however, are based on small samples of tissue, which are dissected and afterwards treated with digestive enzymes. Moreover, this is usually not performed with clinical grade products. This makes these strategies less suitable for direct clinical translation where large quantities of clinical-grade cells are necessary.

Here we show a novel isolation method of human kPSCs for whole organs based on perfusion with clinical grade enzymes and materials. The protocol is adapted from the clinical islets of Langerhans isolation protocol currently in use for clinical application in our center ${ }^{18}$.

This is the first clinical grade method where large quantities of kPSCs can be achieved. The variability in cell yield is largely donor dependent. However, when the cell yield we currently obtain from a fraction of the crude cell suspension of three different donors is extrapolated, in theory, an average yield of $2.7 \times 10^{12} \mathrm{kPSCs}$ per donor could be achieved. As MSC therapy typically consists of 2 cell infusions with $1-2 \times 10^{6}$ cells $/ \mathrm{kg}$ body weight ${ }^{4}$, these cell numbers are sufficient for allogenic treatment of several patients.

One critical step in the isolation procedure is the duration of collagenase digestion. When the digestion period is too short, large clumps of tissue will remain, which will be harder to culture. When the perfusion period is too long, increased cell death may be observed. Therefore, as soon as the kidney starts to become soft and the fluids less transparent, the kidney should be massaged gently and the collagenase treatment should be stopped.

Another critical step is the culture of the cells after NG2 cell enrichment. Sometimes after the NG2 cell enrichment, the perivascular cells do not start to proliferate or start to grow in 3D structures. In this case, when the cells are trypsinized and reseeded, the cells will usually start to grow in monolayer culture.

As starting material, human transplant grade kidneys discarded mainly for surgical reasons were used. These are functional organs without major fibrosis. We acknowledge that this might be a limitation as this is a relatively rare and hard to obtain organ source. Explanted kidneys 
might be another source; however, depending on the reason of kidney explantation, these kidneys might contain more fibrosis and thus myofibroblasts, and therefore care should be taken since myofibroblasts might be isolated and cultured instead of perivascular cells.

As the kPSCs isolated with this protocol show organo-typic properties with kidney epithelial wound healing capacities ${ }^{15}$, cell therapy with kPSCs in kidney diseases and transplantation would be an interesting future application. For this purpose, there are several strategies of cell/cell product delivery. The first strategy is IV infusion of KPSCs, as used currently in the bmMSC clinical studies. Another interesting application is the use of kPSCs or kPSC-excreted factors in machine perfusion before transplantation. In this way, the quality of the explanted kidney might improve which could lead to improved kidney function after transplantation. For both strategies, kPSCs are an interesting new cell source to further explore for clinical purposes.

\section{Disclosures}

D.G.L., M.A.E., and T.J.R. are associated with a patent application regarding this research. The other authors have nothing to disclose.

\section{Acknowledgements}

This research has received funding from the European Community's Seventh Framework Program (FP7/2007-2013) under grant agreement number 305436 STELLAR.

\section{References}

1. Friedenstein, A. J., et al. Precursors for fibroblasts in different populations of hematopoietic cells as detected by the in vitro colony assay method. Exp Hematol. 2 (2), 83-92 (1974).

2. Dominici, M., et al. Minimal criteria for defining multipotent mesenchymal stromal cells. The International Society for Cellular Therapy position statement. Cytotherapy. 8 (4), 315-317 (2006).

3. Pittenger, M. F., et al. Multilineage potential of adult human mesenchymal stem cells. Science. 284 (5411), 143-147 (1999).

4. Leuning, D. G., Reinders, M. E., de Fijter, J. W., Rabelink, T. J. Clinical translation of multipotent mesenchymal stromal cells in transplantation. Semin Nephrol. 34 (4), 351-364, (2014).

5. Reinders, M. E., et al. Autologous bone marrow-derived mesenchymal stromal cells for the treatment of allograft rejection after renal transplantation: results of a phase I study. Stem Cells Transl Med. 2 (2), 107-111 (2013).

6. Perico, N., et al. Autologous mesenchymal stromal cells and kidney transplantation: a pilot study of safety and clinical feasibility. Clin $J$ Am Soc Nephrol. 6 (2), 412-422 (2011).

7. Perico, N., et al. Mesenchymal stromal cells and kidney transplantation: pretransplant infusion protects from graft dysfunction while fostering immunoregulation. Transpl Int. 26 (9), 867-878, (2013).

8. Tan, J., et al. Induction therapy with autologous mesenchymal stem cells in living-related kidney transplants: a randomized controlled trial. JAMA. 307 (11), 1169-1177, (2012).

9. Crisan, M., et al. A perivascular origin for mesenchymal stem cells in multiple human organs. Cell Stem Cell. 3 (3), $301-313$ (2008).

10. Sacchetti, B., et al. No Identical "Mesenchymal Stem Cells" at Different Times and Sites: Human Committed Progenitors of Distinct Origin and Differentiation Potential Are Incorporated as Adventitial Cells in Microvessels. Stem Cell Reports. 6 (6), 897-913, (2016).

11. Chen, W. C., et al. Human myocardial pericytes: multipotent mesodermal precursors exhibiting cardiac specificity. Stem Cells. 33 (2), 557-573, (2015)

12. Dekel, B., et al. Isolation and characterization of nontubular sca-1+lin- multipotent stem/progenitor cells from adult mouse kidney. $J$ Am Soc Nephrol. 17 (12), 3300-3314, (2006).

13. Li, J., et al. Collecting duct-derived cells display mesenchymal stem cell properties and retain selective in vitro and in vivo epithelial capacity J Am Soc Nephrol. 26 (1), 81-94, (2015).

14. Pelekanos, R. A., et al. Comprehensive transcriptome and immunophenotype analysis of renal and cardiac MSC-like populations supports strong congruence with bone marrow MSC despite maintenance of distinct identities. Stem Cell Res. 8 (1), 58-73, (2012).

15. Leuning, D. G., et al. Clinical-Grade Isolated Human Kidney Perivascular Stromal Cells as an Organotypic Cell Source for Kidney Regenerative Medicine. Stem Cells Transl Med. (2016).

16. Bruno, S., et al. Isolation and characterization of resident mesenchymal stem cells in human glomeruli. Stem Cells Dev. 18 (6), 867-880, (2009).

17. Ryan, M. J., et al. HK-2: an immortalized proximal tubule epithelial cell line from normal adult human kidney. Kidney Int. 45 (1), $48-57$ (1994).

18. Nijhoff, M. F., et al. Glycemic Stability Through Islet-After-Kidney Transplantation Using an Alemtuzumab-Based Induction Regimen and Long-Term Triple-Maintenance Immunosuppression. Am J Transplant. (2015). 